\title{
The effects of stimulus alignment on children's performance in a conservation of length problem*
}

\author{
LAUREN HARRIS and TERRY ALLEN \\ Michigan State University, East Lansing, Mich. 48823
}

In the usual conservation-of-length task, one of two horizontally aligned sticks is displaced to the left and $S$ is asked whether or not the sticks, previously judged equal in length, are now unequal. On the possibility that this procedure confounds a left-right discrimination task with the conservation task per se, 18 nonconserving first-grade children were each given four trials with the sticks in horizontal alignment and four in vertical alignment. Contrary to prediction, the incidence of conservation judgments did not increase in the vertical alignment condition, though speed of judgment increased slightly.

Piaget (1928) asked 4- to 12-year-old children to describe the spatial relationships among three objects in a row: a pencil to the left, matches in the middle, and a coin to the right. The 9- to 11-year-olds could say that the pencil was left, the coin right, and the matches in the middle-in the absolute sense-but only the 11 - to 12-year-olds could grasp that the matches were simultaneously to the right in relation to the pencil and to the left in relation to the coin. Before 11 years of age, Piaget argued, the child does not realize ". . that an object must necessarily be to the right or left of something ... but thinks of all these relations as existing in themselves, absolutely [p. 131]." Failure, Piaget argued, represents the absence of the concept of a relation.

In a recent study (Harris, 1969), this view was challenged as an explanation of left-right discrimination. Children in kindergarten and second-grade were asked the same series of questions about the array of objects aligned horizontally, as well as the identical array aligned vertically (i.e., with a pencil above, matches in the middle, and penny below). Thus, in the up-down test, the logic of relation required to recognize that the matches in the middle were both below the pencil and above the penny was the same as the logic in the left-right test required to recognize that the matches were both to the right of the pencil and to the left of the penny. The results were clear: All children, even the youngest, described the spatial relationships accurately so long as the objects were aligned vertically, but

*Thanks are due Suzanne Marshall, for her help in constructing the apparatus and testing Ss, and the staff and children of the Red Cedar Elementary School, East Lansing, Michigan, for their cooperation. failed when they were aligned horizontally. They understood the concept of a relation but, when faced with a horizontal array, became confused because they had not yet mastered basic left-right discrimination.

The following question arises: Do tests of other concepts of logical relationships similarly include a left-right component? The conservation of length task seemed a reasonable candidate for study, since assessment (e.g., Piaget et al, 1960; Gelman, 1969; Kingsley \& Hall, 1967) typically is by means of questions about the relative lengths of two horizontally aligned sticks after one stick has been displaced (see Fig. 1). Since the displaced stick is moved either to the child's left or right, the problem posed to the child might involve a spatial rather than, or in addition to, a logical dimension. In other words, after displacement (e.g., Stick CD moved to left), the relative positions of the two sticks change in that $D$ now is simultaneously to the left of $B$ and to the right of $A$. The situation seems similar to that posed in the relational questions about the three-object array in the studies previously described-questions which, when concerned with the spatial dimension left-right, are particularly difficult for young children. Thus, the nonconserver's failure could stem from his confusion about left and right at Point D, and this confusion may disrupt his ability to judge that the gain at one end is exactly balanced by a loss at the other.

The results of the aforementioned study (Harris, 1969) suggested an analogous test on the conservation problem. Accordingly, conservation of length was assessed with the sticks in the usual horizontal alignment and compared, within each child, with performance with the same sticks in vertical alignment, i.e., in a spatial alignment (up-down) in which children of the age tested are known to be considerably more skilled. If the horizontal alignment, in fact, incorporates a left-right discrimination problem, vertical alignment should improve performance (i.e.. more conservation judgments should be made). In other words, in this situation, after Stick CD is displaced down (Fig. 1), the relative positions of the two sticks change, in that $D$ now is simultaneously below $B$ and above $A$. But in the test of up-down discrimination (Harris, 1969), this kind of situation posed no problems, as we have said, even to the youngest children.

In addition to the basic tests for conservation of length, response latencies were measured to see if the presumed lesser difficulty of the vertical condition would be reflected in faster judgments.

\section{SUBJECTS}

Since conservation of length is ordinarily assessed with the materials aligned horizontally, the use of conservers to test the difference between the vertical and horizontal alignments would be inappropriate, since it is improbable that a child, having demonstrated conservation "horizontally," would fail "vertically." Ideally, the Ss should be nonconservers in or near a transitional stage and old enough to understand the instructions. Since previous research (e.g., Piaget et al, 1960) finds that about 7 years of àge usually marks the period of acquisition of conservation of length, slightly younger children were chosen for the current study. The Ss were 18 first-graders (nine boys). The average age of the boys was 6 years, 10 months (range: 6:3-7:2) and of the girls, 6 years, 9 months $(6: 3-7: 2)$.

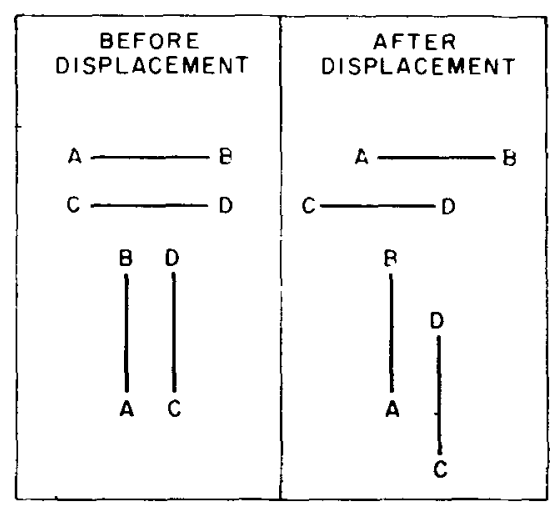

Fig. 1. Position of sticks before and after displacement when aligned horizontally (top) and when aligned vertically (bottom). 


\section{MATERIALS}

The pretest materials were a red pencil, 7 in. long, a purple pencil, $51 / 2$ in. long, and two $2 \times 1 \frac{11 / 2}{} \times 7 / 8$ in. blocks of wood. The test materials were $102 \times 3 / 8 \mathrm{in}$. wood sections, five red, five green (to be placed end to end to make two 10-in.-long sticks). Each section had a light magnet embedded in its long side. The test board was a 12-in. gray metal square, mcunted perpendicular to the table.

EXPERIMENTAL DESIGN

Dach child received eight trials, four with the sticks in vertical alignment and four in horizontal alignment. In both alignm ?nt conditions, trials were presented in a random sequence, with the constraint that the same alignment appeared, at most, twice in succession. Each alignment condition included two trials with Transformation 1 and two with Transformation 2. The two trials within each transformation condition differed only in respect to the stick that was changed.

In Transformation 1, the changed stick was moved in a straight line so as to project beyond the other; in Transformation 2 , the changed stick was bent $(\Omega)$ so that the other stick projected beyond it. The position and color of the changed stick varied randomly, with the constraint that on four trials it was on the right (in vertical alignment) or on top (in horizontal alignment), and on four trials on the left or bottom. Finally, on four tests (two within each alignment condition), the red stick was changed, and on four, the green stick was changed.

\section{PROCEDURE}

Each child came individually to the testing room, a spare room in the school. He sat at a table directly before the test apparatus, and $\mathrm{E}$ sat to his left. A tape recorder was on the table to the child's left.

\section{Pretest}

The $E$ placed the two pencils on the table parallel to each other and approximately $1 \mathrm{in}$. apart, the long sides facing the child, and asked, "Is the purple pencil just as long as the red pencil?" [Waited for reply.] "Is one pencil longer than the other?" [Waited for reply.] The $E$ then placed the blocks on the table parallel to each other, approximately $1 \mathrm{in}$. apart, so that one block did not project beyond the other and repeated the same two questions.

The pretest questions assessed each child's understanding of the concepts of differentness (longer than) and sameness (just as long as). All children answered these questions correctly and unhesitatingly.

Other than the substitution of length for number and the use of a pretest, the test procedure was identical to Rothenberg's (1969). Basically, Rothenberg's procedure requires the child to reply both positively and negatively to be classified as a conserver (or nonconserver). The procedure thus prevents the misclassification of a child as a conserver on the basis of response set.

The $E$ arranged the red and green sections into two 10 -in.-long sticks on the test board, each stick parallel to the other and 2 in. apart, so that one did not project beyond the other. E asked, "Is the red stick just as long as the green stick?" If S said no, he was asked to align the sticks until he judged them to be of equal length. All children either immediately judged or quickly came to judge the sticks as equal in length. If $S$ said yes, then $E$ performed the first transformation on the sticks and asked the first two test questions: "Is the red stick just as long as the green stick?" and "Is one stick longer than the other?" If $S$ answered no to the first question and yes to the second (nonconservation), he was asked which stick was longer, why it was longer, and, finally, how he could make them the same again. If he answered yes to the first question and no to the second (conservation), he was asked to explain his answer. These three questions comprised one "trial."

The combination of either negative or positive replies to both questions would reflect lack of understanding of the questions. These combinations never occurred, presumably because they were precluded by the pretest.

After each transformation and set of questions, E returned the sticks to their original positions of equivalence and then reassessed S's judgment by repeating the first question, "Is the red stick as long as the green stick?" The next transformation was not presented until $S$ had made the judgment of equivalence.

Complete testing took about $25 \mathrm{~min}$ for each child.

\section{SCORING}

\section{Conservation Judgments}

Rothenberg's (1967) scoring procedure was followed exactly. The two trials on Transformation 1 (differing only in respect to the stick that was changed) comprised a single "conservation assessment task." A child whose responses classified him as a conserver on this task received a score of 7. A perfect conservation score would be $7 \times 4$ conservation tasks $=28$ points. A perfect nonconservation score would be 2 (a minimum score awarded on each task for understanding the instructions) $\times 4$ tasks $=8$ points.

\section{Response Latencies}

Response latencies, defined as time between the end of E's question and S's reply, were obtained by stopwatch from the tape recording of S's answers. Timings were made in ignorance of the alignment condition for any particular trial. The analysis treated each $\mathrm{S}$ as his own control by expressing each of his scores in terms of its deviation from his own average score for all 16 questions comprising his eight trials. The analysis thus compared, within each $S$, latencies on the vertical alignment trials with latencies on the horizontal trials in terms of each S's average for all trials. The resulting deviation scores, with a constant added, were analyzed in a mixed-design 2 ( $\operatorname{sex}$ of $S$ ) by 2 (alignment condition) by 16 (questions) analysis of variance.

\section{RESULTS AND DISCUSSION}

On the horizontal alignment trials, 14 of the 18 Ss ( 7 boys, 7 girls) were classifiable as "perfect" nonconservers, one (boy) as a "perfect" conserver, and three ( 1 boy, 2 girls) as transition stage. On the vertical alignment trials, however, the scores failed to improve. Indeed, each S's vertical score was identical to his horizontal score, irrespective of his classification. There were no sex differences.

Every S, on the great majority of questions, replied very quickly. The range was $0.5-18 \mathrm{sec}$, with $80 \%$ of all latencies $2 \mathrm{sec}$ or less and only $2 \%$ more than $5 \mathrm{sec}$. Though the latencies in the horizontal alignment were slower, the difference was insignificant $(F<1.0)$ in the analysis of variance of deviation scores. Further examination of individual Ss' scores disclosed 13 of 18 (seven boys, six girls) with longer average latencies for horizontal than vertical. These 13 Ss included the one conserver. This proportion, on the binomial expansion test, exceeded a chance level $(Q=P=0.5, p<.015)$. In each case, however, the actual difference was less than 1 sec.

The very high incidence of nonconservation with the sticks in the usual horizontal alignment indicates that the choice of first-graders for this study was appropriate. The absence of sex differences is also consistent with previous research. The complete lack of any improvement in accuracy of judgment when the sticks were placed vertically seems to preclude the operation of left-right confusion as an important component in the conservation-of-length task. Therefore, even though stimulus alignment has exercised a substantial effect in studies of other perceptual-cognitive behaviors (Harris, 1969; Howard \& Templeton, 1966), it now seems that previous studies' failure to control for alignment in the assessment of conservation of length has not distorted or otherwise obscured the 
nature of this particular phenomenon. Whether the child will conserve or not seems quite independent of the particular way in which the stimulus materials are aligned.

The results of analyses of the individual response latency scores, however, suggest that alignment may have a small effect, not on the judgments themselves, but simply on the ease with which the information in the task can be processed, irrespective of the use to which it is put. Or to make the distinction another way, imagine a child just learning how to add but still lacking the rule for "carrying" numbers being able to add a series of numbers faster when they are arranged in a column than in a row but arriving at an incorrect sum in either case. The vertical alignment lets him add more quickly but not more accurately. For accuracy, he needs an additional principle.

\section{REFERENCES}

GELMAN, R. Conservation acquisition: $A$ problem of learning to attend to relevant attributes. Journal of Experimental Child Psychology, 1969, 7, 167-187.

HARRIS, L. Discrimination of left-right directionality and development of the logic of relations. Paper presented at the Biennial Meetings of the Society for Research in Child Development, Santa Monica, Calif.. March 29, 1969.

HOWARD, I. P., \& TEMPLETON, W. B. Human spatial orientation. London: Wiley, 1966.

KINGSLEY, R. C., \& HALL, V. C. Training of conservation through the use of learning sets. Child Development, 1967 , $38,1111-126$.

PIAGET, J. Judgment and reasoning in the child. New York: Harcourt, Brace, 1928.

PIAGET, J.. INHELDER, B., \& SZEMINSKA, A. The child's conception of geometry. New York: Basic Books, 1960.

ROTHENBERG, B. B. Conservation of number among four- and five-year-old children: Some methodological considerations. Child Development, 1969, $40,383-406$. present experiment studied the performance of H-MS and L-MS groups in a paired-associate (PA) task under two levels of formal intralist response similarity (IRS). It was of particular interest to see how Ss with different MS dealt with the unequal degree of interference present in the lists.

\section{SUBJECTS}

A total of 298 female introductory psychology students at Mary Washington College took a MS test consisting of one-trial free recall of 20 two-digit numbers followed by the free recall of 20 adjective-noun combinations. These items were presented on a Kodak Carousel 800 slide projector at a 2-sec rate. Detailed instructions and procedures in administering and scoring the MS test are presented elsewhere (Furukawa, Suydam, \& Miller, 1969). All students were rank ordered for items recalled, and the $\mathbf{4 0}$ highest and lowest scoring students formed, respectively, the H-MS and L-MS groups. Scores on the MS pretest ranged from 11.00 to 14.25 for $H$-MS $\mathrm{Ss}$ and from 4.25 to 7.50 for L-MS Ss. The minimum H-MS score and the maximum L-MS score compare favorably with those for equivalent groups reported by Furukawa et al. Approximately 2 weeks following the MS pretest, the Ss in each group were individually administered a PA list.

\section{LISTS}

Two CVC trigram PA lists, high in meaningfulness $(M=95 \%)$, were constructed from Archer's (1960) norms. The stimuli, which were common to both lists, were PET, CUP, BIN, LAB, FOR, WIG, DAY, and HOW. The lists differed from each other in terms of the degree of IRS. The high IRS items, respectively paired with the above stimuli, were SAG, SAM, MAG, PAM, MAS, PAS, GAP, and GAM. The low IRS items were DUG, FEW, JUD, VAT, HIS, ROX, BAM, and PIK. Lists were presented on a Stowe memory drum (Model 459B) at a 2:2-sec rate with a 4 -sec intertrial interval. Four different orders of each list were generated in order to minimize serial learning.

\section{DESIGN}

Four experimental groups $(\mathrm{N}=20)$ were formed: $\mathrm{HH}, \mathrm{HL}, \mathrm{LL}, \mathrm{LH}$. The first letter refers to S's high $(\mathrm{H})$ or low (L) ability to recall, as indexed by her score on the MS pretest. The second letter refers to the IRS list (high, $\mathrm{H}$; low, L) to which $\mathrm{S}$ was exposed. All $\mathrm{H}$ and L-MS Ss were assigned randomly to a particular IRS condition. Each $\mathbf{E}$ ran one-half of the Ss in each of the four experimental conditions.

\section{PROCEDURE}

Each $S$ was read a standard set of PA learning instructions. In order to
* The authors are indebted to Evelyn
Grant and Franceen Huddleston for as the Es in this investigation. found that high memory span (H-MS) Ss performed significantly better than low memory span (L-MS) Ss on a serial learning task consisting of $100 \%$ association-value CVC trigrams. The 